\title{
DNA mismatch repair and TP53 defects are early events in uterine carcinosarcoma tumorigenesis
}

\author{
Nicholas P Taylor ${ }^{1}$, Israel Zighelboim ${ }^{1}$, Phyllis C Huettner ${ }^{2}$, Matthew A Powell ${ }^{1}$, \\ Randall K Gibb ${ }^{1}$, Janet S Rader ${ }^{1}$, David G Mutch ${ }^{1}$, Tina B Edmonston ${ }^{3}$ \\ and Paul J Goodfellow ${ }^{4}$
}

${ }^{1}$ Division of Gynecologic Oncology, Washington University School of Medicine and Siteman Cancer Center, St Louis, MO, USA; ${ }^{2}$ Department of Pathology and Immunology, Washington University School of Medicine and Siteman Cancer Center, St Louis, MO, USA; ${ }^{3}$ Department of Pathology, Anatomy, and Cell Biology, Thomas Jefferson University, Philadelphia, PA, USA and ${ }^{4}$ Department of Surgery and Division of Gynecologic Oncology, Washington University School of Medicine and Siteman Cancer Center,

St Louis, MO, USA

\begin{abstract}
Growing molecular evidence shows that uterine carcinosarcomas are clonal tumors. The carcinoma component has a dominant effect in the aggressive clinical behavior of these tumors. Defective DNA mismatch repair affects up to $30 \%$ of endometrial adenocarcinomas. The frequency and importance of defective DNA mismatch repair in the histiogenesis of uterine carcinosarcomas remains controversial. We studied the pattern and frequency of defective DNA mismatch repair and TP53 alterations in the epithelial and mesenchymal components of 28 uterine carcinosarcomas. We found evidence of defective DNA mismatch repair in six cases $(21 \%)$ with a concordance rate of $83 \%$ for carcinoma-sarcoma pairs $(\kappa=0.887, P<0.001)$. Lack of immunostaining for the MLH1 protein was demonstrated in both components in two of these tumors. TP53 defects were evaluated by 17 p deletion analysis and p53 immunostaining. Nineteen carcinoma (68\%) and 18 sarcoma (64\%) components had evidence of either TP53 allelic loss or p53 overexpression. These defects proved clonal in $76 \%$ of cases $(\kappa=0.602, P=0.003)$. Our results indicate that defective DNA mismatch repair and TP53 defects are common early events in carcinosarcoma tumorigenesis. The high rate of concordance for these molecular defects between the carcinoma and sarcoma components adds to existing molecular evidence that carcinosarcomas are clonal malignancies.
\end{abstract}

Modern Pathology (2006) 19, 1333-1338. doi:10.1038/modpathol.3800654; published online 30 June 2006

Keywords: carcinosarcoma; malignant mixed müllerian tumor; microsatellite instability; DNA mismatch repair; TP53; clonality

Carcinosarcomas of the uterus account for $1-2 \%$ of all malignancies of the uterine corpus. The pathologic hallmark of these tumors is the presence of malignant epithelial and mesenchymal components. These mixed malignancies can affect any of the müllerian derivatives of the female reproductive tract. Despite their relatively low incidence, these aggressive tumors represent a significant clinical problem. ${ }^{1}$ The overall survival at 5 years for all patients with cancer of the uterine corpus is $84 \%$ and only $31 \%$ for patients with a uterine carcinosarcoma. ${ }^{2,3}$

Correspondence: Dr NP Taylor, MD, Division of Gynecologic Oncology, Washington University School of Medicine, 4911 Barnes-Jewish Hospital Plaza, Maternity Building, 3rd Floor, St Louis, MO 63110, USA.

E-mail: taylorni@msnotes.wustl.edu

Received 17 May 2006; revised 31 May 2006; accepted 1 June 2006; published online 30 June 2006
There is clinical and molecular evidence suggesting that uterine carcinosarcomas are clonal malignancies. ${ }^{4-10}$ Immunohistochemical studies of carcinosarcomas have suggested a common epithelial origin. ${ }^{4}$ Studies in nude mice have demonstrated that carcinoma cells derived from a carcinosarcoma cell line can give rise to tumors that include both epithelial and mesenchymal components whereas sarcoma cells do not. ${ }^{5}$ Furthermore, the epithelial and mesenchymal components frequently share patterns of X-inactivation, allelic loss, and TP53 mutation. ${ }^{6,7}$ Clinically, the carcinoma component is more frequently found in metastatic deposits, ${ }^{4,8}$ leading most clinicians to approach this tumor as a poorly differentiated carcinoma rather than a sarcoma.

Defective DNA mismatch repair is a relatively common phenomenon in endometrial adenocarci- 
nomas, affecting more than $25 \%$ of these tumors. ${ }^{11}$ The microsatellite instability (MSI) tumor phenotype is a hallmark of defective DNA mismatch repair. Although MSI has been reported to be infrequent in uterine carcinosarcomas $(\sim 5 \%),{ }^{12,13}$ recent studies from our laboratory have suggested that MSI may be more common in uterine carcinosarcomas than previously described and that defective DNA mismatch repair may be a feature unique to the epithelial component of these tumors. ${ }^{12-14}$

We therefore sought to determine the DNA mismatch repair and TP53 status in each component of uterine carcinosarcomas in an attempt to better understand the histiogenesis of these malignancies.

\section{Materials and methods}

\section{Patient Population, Tissues and DNA Specimens}

This study was approved by The Washington University Medical Center Human Studies Committee and all participants explicitly consented to participate in ongoing molecular studies. Tumor and blood samples were collected from these patients and demographic and clinical information was entered in a computerized database. Of the patients consented between 1993 and 2004, there were 28 with a pathologic diagnosis of uterine carcinosarcoma for whom archival tissue blocks and slides were available for review when this study was initiated. The mean age for these patients was $72 \pm 10$ years (age \pm s.d.). Twenty-one cases $(75 \%)$ were Caucasian, six were African-American $(21 \%)$ and no race was available in one case. Histologic sub-type of the individual components and stage distribution are presented in Table 1.

Diagnostic slides were reviewed and the histopathologic classification of the cases confirmed by a single gynecologic pathologist (PCH). Areas of high neoplastic cellularity ( $\geq 70 \%)$ for carcinoma and sarcoma elements were identified and marked for tissue dissection and DNA extraction. Laser capture micro-dissection and/or needle dissection of mesenchymal and epithelial components of each tumor was performed from unstained $5 \mu \mathrm{m}$ slides. Tissue was placed in $50 \mu \mathrm{l}$ of LCM buffer $(0.04 \%$ Proteinase $\mathrm{K}$ in $1 \mathrm{mM}$ EDTA, $10 \mathrm{mM}$ Tris $\mathrm{HCl}$ and $1 \%$ Tween $20(\mathrm{pH} 8.0))$ and digested overnight at $37^{\circ} \mathrm{C}$ in a rotating incubator. The proteinase $\mathrm{K}$ was then inactivated at $95^{\circ} \mathrm{C}$ for $8 \mathrm{~min}$. This DNA stock solution was then used for PCR amplification.

\section{MSI Analysis}

MSI analysis of the individual epithelial and sarcomatous components was performed as previously described ${ }^{15,16}$ using five National Cancer Institute consensus microsatellite markers. ${ }^{17}$ Each component was evaluated individually and confirmatory MSI typings were performed up to three times as needed. Tumor components were desig-
Table 1 Histology and stage distribution of uterine carcinosarcoma cases

\begin{tabular}{lc}
\hline & $\mathrm{N}(\%)$ \\
\hline Carcinoma & \\
$\quad$ Serous & $13(46 \%)$ \\
Endometrioid & $11(39 \%)$ \\
Serous and endometrioid & $3(11 \%)$ \\
Undifferentiated & $1(4 \%)$ \\
& \\
Sarcoma & \\
Leiomyosarcoma & $10(36 \%)$ \\
Undifferentiated & $8(29 \%)$ \\
Rhabdomyosarcoma & $4(14 \%)$ \\
Chondrosarcoma & $2(7 \%)$ \\
Mixed & $4(14 \%)$ \\
Stage & \\
I & \\
II & $11(39 \%)$ \\
III & $2(7 \%)$ \\
IV & $7(25 \%)$ \\
\end{tabular}

nated as having high-level MSI (MSI-H) if novel PCR bands were present in at least two of the five consensus panel markers, low-level MSI (MSI-L) if a single marker demonstrated MSI or microsatellite stable if there was no evidence of MSI in any marker. Only MSI-H was considered diagnostic of defective DNA mismatch repair.

\section{Immunohistochemistry for MLH1, MSH2 and MSH6}

Immunohistochemistry for MLH1, MSH2 and MSH6 was performed using $5 \mu$ m-thick paraffin sections mounted on charged slides and interpreted by an experienced pathologist (TBE). Specific antibodies were processed as previously described using the following concentrations: 1:200 for MLH1 (Clone G168-728; BD PharMingen, San Diego, CA, USA), 1:400 for MSH2 (Clone FE11; Zymed Laboratories, San Francisco, CA, USA) and 1:600 for MSH6 (Clone 44; BD Transduction Laboratories, San Diego, CA, USA).$^{18}$ Nuclear staining was read as positive and absence of nuclear staining was read as negative.

\section{Evaluation of TP53 Status: Loss of Heterozygosity for $17 p$ and p53 Immunostaining}

The status of TP53 was evaluated indirectly by loss of heterozygosity (LOH) analysis and p53 immunohistochemistry. LOH analysis was performed using the consensus MSI marker D17S250, five previously described SNPs located within $200 \mathrm{~Kb}$ of this marker (rs12602312, rs6503741, rs575809, rs4795339, rs1014263) analyzed by restriction digest (primers, amplification conditions as well as restriction endonucleases available upon request), and a newly designed dinucleotide repeat microsatellite marker 23CA (forward primer-TCTTGGCACATCTGAAA GCA, reverse primer-GTAACCGGCTGTGCTGTC TC, Tm: $60^{\circ} \mathrm{C}$ ). 
Immunostains for p53 were performed on $5 \mu \mathrm{m}$ thick sections using clone 1803 (BioGenex, San Ramon, CA, USA) at a dilution of 1:200 using pretreatment and staining with the Ventana BenchMark XT IHC/ISH Staining Module (Ventana Medical Systems Inc., Tuscon, AZ, USA) following manufacturers' recommended protocols. IHC results were interpreted for the individual components of each tumor by an experienced gynecologic pathologist (PCH). p53 IHC was considered positive when tumor cells demonstrated strong nuclear staining.

$\mathrm{LOH}$ for $17 \mathrm{p}$ or TP53 overexpression (as determined by immunostaining) was considered evidence of a TP53 defect.

\section{Statistical Analysis}

Descriptive statistics were used to evaluate cohort, disease and molecular characteristics. Fisher's exact test and kappa test were used to evaluate associations and concordance. A $P$-value of $<0.05$ was considered significant.

\section{Results}

\section{Defective DNA Mismatch Repair and TP53 Defects in Carcinoma and Sarcoma Pairs}

Six out of 28 carcinoma-sarcoma pairs (21\%) had evidence of defective DNA mismatch repair based on the MSI-H phenotype in at least one component (carcinoma or sarcoma). Twenty-one out of 28 pairs (75\%) had suspected TP53 defects as evidenced by either loss of heterozygosity for $17 p$ (15/28 cases) or by p53 overexpression (15/26 cases) in either component. The majority of cases in our series $(79 \%)$ were informative for clonality analysis. The MSI and TP53 status for the entire cohort is presented in Table 2 .

\section{Defective DNA Mismatch Repair in Carcinosarcomas}

Five out of six tumors (83\%) showed concordance for the MSI-H phenotype in both epithelial and mesenchymal components $(\kappa=0.887, P<0.001)$. Lack of immunostaining for the MLH1 protein was demonstrated in both components in two of these tumors (cases 1144 and 1689, Figure 1a). In four cases MLH1, MSH2 and MSH6 immunostains were normal, despite phenotypic evidence of defective DNA mismatch repair (MSI-H). There was no difference in the frequency of MSI-H between the carcinoma (5/28) and sarcoma (6/28) components.

\section{7p LOH and p53 Overexpression}

$17 \mathrm{p} \mathrm{LOH}$ was identified in at least one component of the tumor in 15 cases (54\%). Specifically, LOH occurred in 12 of $27(44 \%)$ carcinomas (it was not possible to determine $17 \mathrm{p}$ status in one carcinoma specimen-case 160) and 14 of $28(50 \%)$ sarcomas. Most carcinoma-sarcoma pairs $(73 \%)$ had identical LOH patterns and as such, were considered clonal for $17 \mathrm{p}$ deletion $(\kappa=0.705, P<0.001)$.

p53 immunostaining was successful for 26 tumors. Overall, 15 cases (58\%) demonstrated p53 overexpression. Fourteen carcinoma (54\%) and 13 sarcoma components (50\%) showed p53 overexpression. There was $80 \%$ concordance for p53 overexpression in the carcinoma-sarcoma pairs $(\kappa=0.769$, $P<0.001)$

Taken together, the findings for $17 \mathrm{p} \mathrm{LOH}$ and IHC suggest TP53 defects in at least 19 carcinomas $(68 \%)$ and 18 sarcomas (64\%). In most cases (75\%), 17p $\mathrm{LOH}$ and TP53 overexpression were consistent (Figure 1b). However, some cases demonstrated discordant p53 immunostaining despite clonal evidence of 17p LOH or vice versa (Figure 1c).

Overall, TP53 defects proved clonal in $76 \%$ of cases $(\kappa=0.602, P=0.003)$.

\section{Discussion}

Previous molecular and clinical studies have suggested that uterine carcinosarcomas are clonal malignancies; ${ }^{4-7}$ however, the roles defective DNA mismatch repair and TP53 defects play in these

Table 2 MSI phenotype and TP53 defects in carcinosarcomas

\begin{tabular}{|c|c|c|c|c|}
\hline \multirow[t]{2}{*}{ Case } & \multicolumn{2}{|c|}{ Carcinoma } & \multicolumn{2}{|c|}{ Sarcoma } \\
\hline & $M S I-H$ & TP53 defect & $M S I-H$ & TP53 defect \\
\hline 165, 1161, 1208, 1239, 1338, 1467, 1469, 1491, 1595, 1596, 1634, 1728, 1765, 1860 & - & + & - & + \\
\hline 1689 & + & - & + & - \\
\hline 1643 & + & + & + & + \\
\hline $160,1210,1354,1461,1630,1850$ & - & - & - & - \\
\hline 1203,1413 & + & + & + & - \\
\hline 1144 & + & - & + & + \\
\hline 1230 & - & + & + & + \\
\hline 1828 & - & + & - & - \\
\hline 1848 & - & - & - & + \\
\hline
\end{tabular}

+: Indicate MSI-high phenotype or TP53 defect by either 17p deletion analysis or immunohistochemical overexpression. 


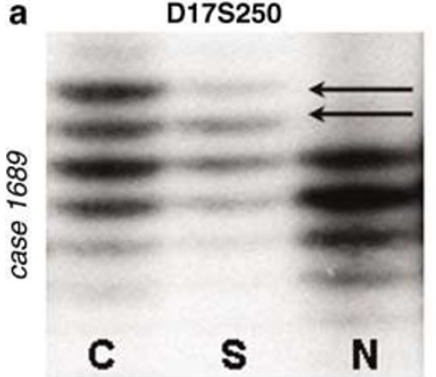

b

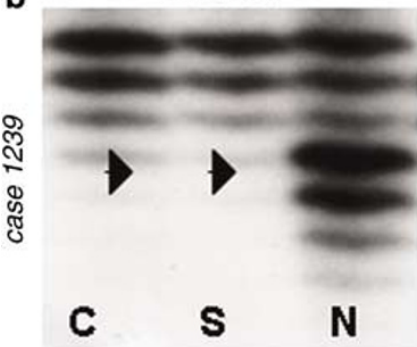

D2S123

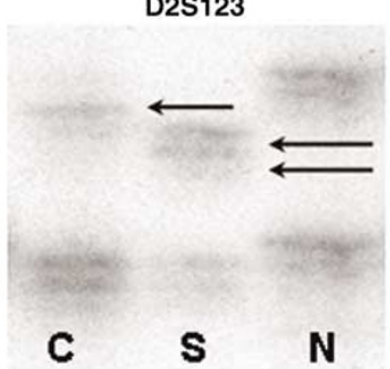

rs12602312

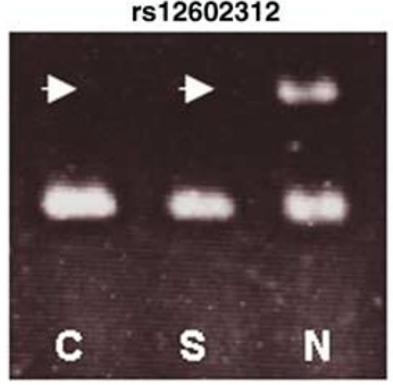

$\alpha-\mathrm{MLH1}$

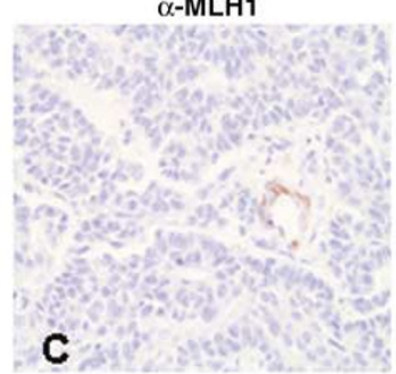

$\alpha-p 53$

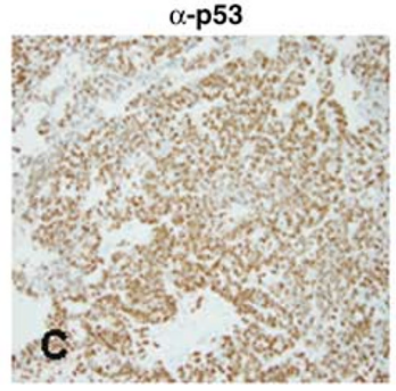

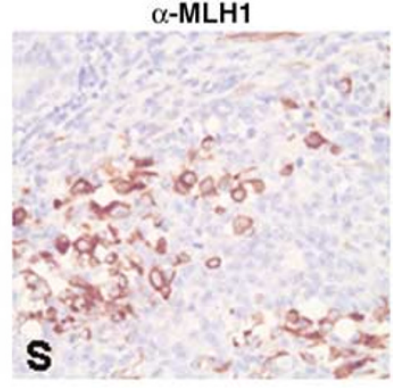

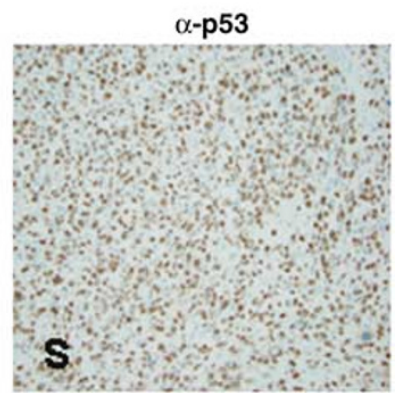

C
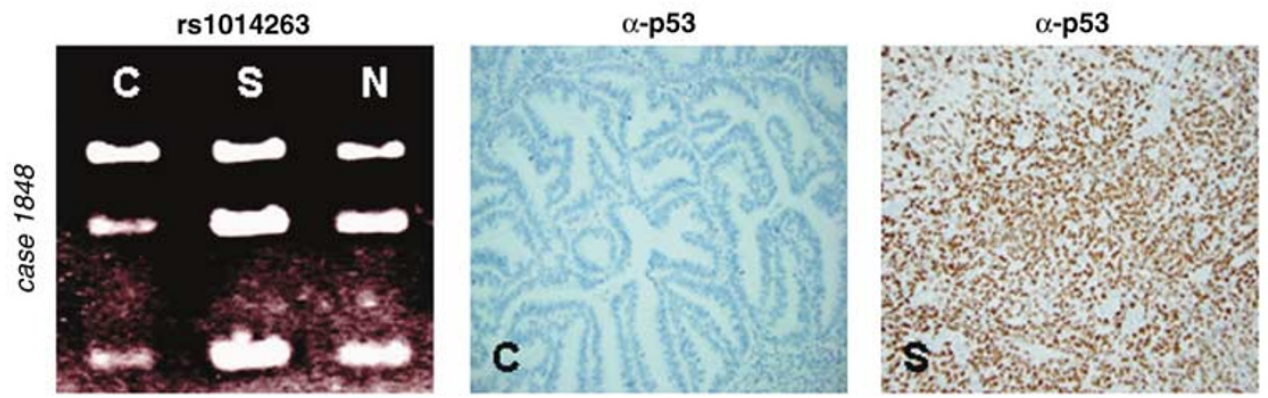

Figure 1 Representative examples of molecular and immunohistochemical findings for carcinosarcomas. (a) Concordant MSI and MLH1 IHC in case 1689. Left two panels show abnormal PCR products characteristic of MSI (arrows) for D17S250 and D2S123 markers in both the epithelial and mesenchymal components. Lack of immunodectable MLH1 protein in the serous carcinoma and chondrosarcoma components of this tumor is illustrated in the right two panels (positive staining in endothelial cells in the sarcomatous element serves as internal positive control). (b) Concordant LOH at D17S250 and rs12602312 (Mae II RFLP) in the carcinomatous and sarcomatous elements of case 1239. Arrowheads indicate alleles lost in the cancer (left two panels). p53 overexpression in the serous carcinoma and mixed (rhabdomyosarcoma and undifferentiated) sarcoma from case 1239 (right two panels). (c) Retention of heterozygosity in both the carcinomatous and sarcomatous elements of case 1848 (left panel). Allelic retention of 17p shown by Cac8I restriction digestion of a 17p SNP (rs1014263). IHC demonstrates p53 overexpression in the leiomyosarcoma but not in the endometrioid carcinoma component (right two panels). C: carcinoma; S: sarcoma; $\alpha$-MLH1: MLH1 antibody; $\alpha$-p53: p53 antibody.

tumors have not been clearly defined. We observed a high overall rate of MSI (21\%), 17p deletion (54\%), and p53 overexpression (58\%) among 28 carcinosarcomas investigated. In our study, both DNA mismatch repair and/or TP53 defects were common to both components of carcinosarcomas. Concordance for the MSI-H phenotype was seen in 5/6 tumors $(83 \%)$, and $76 \%$ of cases proved clonal for TP53 defects as defined by $17 \mathrm{p} \mathrm{LOH}$ or TP53 overexpression. The high degree of concordance of TP53 and/or DNA mismatch repair defects seen in this study provides additional evidence for a clonal origin in these malignancies.

The high frequency with which TP53 and/or DNA mismatch repair defects are shared by the carcinomatous and sarcomatous elements suggest that these lesions occur early in the tumorigenesis (Table 2).
Figure 2 is a schematic representation of where and when TP53 and/or DNA mismatch repair defects occur in the genesis of carcinosarcomas based on our analysis of 28 tumors. In most cases, the progenitor cell that gives rise to both the sarcoma and carcinoma components acquires either a TP53 defect $(14 / 28$ cases; $50 \%)$, loses DNA mismatch repair (case 1689; 4\%) or acquires both defects (case 1643; 4\%). When the initial event occurs in the progenitor cell these defects are shared by the epithelial and mesenchymal tumoral components. Alternatively, the progenitor cell may be affected only by defective DNA mismatch repair (defect present in both descending lineages) and only one compartment subsequently acquires a TP53 alteration (cases 1144, 1203, 1413; 11\%). TP53 alteration in the progenitor followed by loss of mismatch 


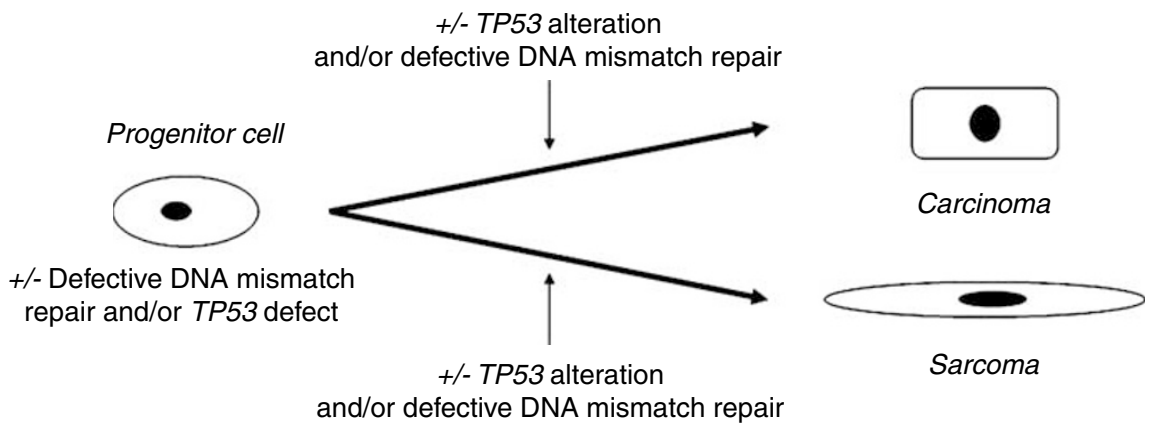

Figure 2 Proposed model for histiogenesis of uterine carcinosarcomas. A normal progenitor cell may acquire a number of genetic mutations including defects in TP53 and DNA mismatch repair. Additional defects are acquired as the tumor differentiates into the carcinoma and sarcoma components. Shared molecular defects (present in the progenitor cell) will be present in both components. Molecular defects acquired later in histiogenesis will be discordant between the two components.

repair in one compartment also occurs (case 1230; $4 \%)$. In some instances, a single cell lineage was affected by either one of these molecular alterations (in case 1828 and in case 1848; 7\%) or no molecular lesion could be identified (6/28 cases; $21 \%)$. In the absence of definitive discordance the case for clonality could still be explained by other molecular mechanisms not explored in our study. Overall, this model supports clonality in at least $71 \%$ of our cohort based on identification of clonal molecular abnormalities.

We found a higher proportion of defective DNA mismatch repair in carcinosarcomas than was previously reported $(21.4 \%$ vs $5 \%) .{ }^{10}$ However, only two of six tumors with MSI-H had defects in MLH1, MSH2, or MSH6 expression based on IHC. This may reflect DNA mismatch repair deficiency attributable to another member of this pathway such as MLH3 or PMS2 or missense changes in one of the proteins that abrogate repair. Alternatively, a novel DNA mismatch repair protein may be involved in carcinosarcoma tumorigenesis. Although unlikely, it is also possible that technical artifacts were responsible for false positive MSI results.

We also found a relatively high rate of p53 overexpression (58\%) in this cohort of carcinosarcomas which is consistent with the range of $30-60 \%$ reported previously for these tumors. ${ }^{19,20}$ p53 overexpression has been associated with biological aggressiveness in endometrioid adenocarcinomas as well as in papillary serous carcinomas of the endometrium. ${ }^{21,22}$ As uterine carcinosarcomas are clinically aggressive malignancies and approximately $54 \%$ of the patients in our cohort had metastatic disease at presentation, it was not surprising to find a high rate of p53 overexpression in these tumors. TP53 overexpression is known to be associated with carcinomas of the serous type. Although 16/28 tumors had serous carcinoma within the epithelial component, there was no association between TP53 defects and the presence of serous carcinoma $(P=0.114)$.

Overall, our data suggest that DNA mismatch repair and TP53 defects are early events in carcino- sarcoma tumorigenesis. Furthermore, the high rate of concordance for these molecular defects among the carcinoma and sarcoma components adds to existing molecular evidence that carcinosarcomas are clonal tumors. Further investigation is needed to determine whether there is an association between DNA mismatch repair and/or TP53 defects and clinical outcomes.

\section{Acknowledgements}

We thank Mary Ann Mallon and Amy Schmidt for their technical support. We would like to thank the Siteman Cancer Center at Washington University School of Medicine and Barnes-Jewish Hospital in St Louis, Missouri, for the use of the Laser Capture Dissection instrument in the Tissue Procurement Core and the Biostatistics Core which provided assistance with the statistical analyses. The Siteman Cancer Center is supported by an NCI Cancer Center Support Grant \#P30 CA91842.

\section{References}

1 Amant F, Cadron I, Fuso L, et al. Endometrial carcinosarcomas have a different prognosis and pattern of spread compared to high-risk epithelial endometrial cancer. Gynecol Oncol 2005;98:274-280.

2 Surveillance, Epidemiology, and End Results (SEER) Program (www.seer.cancer.gov) SEER*Stat Database: Incidence-SEER 9 Regs Public-Use, Nov 2004 Sub (1973-2002), National Cancer Institute, DCCPS, Surveillance Research Program, Cancer Statistics Branch, released April 2005, based on the November 2004 submission.

3 Callister M, Ramondetta LM, Jhingran A, et al. Malignant mixed Mullerian tumors of the uterus: analysis of patterns of failure, prognostic factors, and treatment outcome. Int J Radiat Oncol Biol Phys 2004;58: 786-796.

4 Bitterman P, Chun B, Kurman RJ. The significance of epithelial differentiation in mixed mesodermal tumors 
of the uterus. A clinicopathologic and immunohistochemical study. Am J Surg Pathol 1990;14:317-328.

5 Emoto M, Iwasaki H, Kikuchi M, et al. Characteristics of cloned cells of mixed mullerian tumor of the human uterus. Carcinoma cells showing myogenic differentiation in vitro. Cancer 1993;71:3065-3075.

6 Wada H, Enomoto T, Fujita M, et al. Molecular evidence that most but not all carcinosarcomas of the uterus are combination tumors. Cancer Res 1997;57: 5379-5385

7 Kounelis S, Jones MW, Papadaki H, et al. Carcinosarcomas (malignant mixed mullerian tumors) of the female genital tract: comparative molecular analysis of epithelial and mesenchymal components. Hum Pathol 1998;29:82-87.

8 Silverberg SG, Major FJ, Blessing JA, et al. Carcinosarcoma (malignant mixed mesodermal tumor) of the uterus. A Gynecologic Oncology Group pathologic study of 203 cases. Int J Gynecol Pathol 1990;9:1-19.

9 Abeln EC, Smit VT, Wessels JW, et al. Molecular genetic evidence for the conversion hypothesis of the origin of malignant mixed mullerian tumours. J Pathol 1997;183:424-431.

10 Fujii H, Yoshida M, Gong ZX, et al. Frequent genetic heterogeneity in the clonal evolution of gynecological carcinosarcoma and its influence on phenotypic diversity. Cancer Res 2000;60:114-120.

11 Goodfellow PJ, Buttin BM, Herzog TJ, et al. Prevalence of defective DNA mismatch repair and MSH6 mutation in an unselected series of endometrial cancers. Proc Natl Acad Sci USA 2003;100:5908-5913.

12 Amant F, Dorfling CM, Dreyers L, et al. Microsatellite instability in uterine sarcomas. Int J Gynecol Cancer 2001;11:218-223.

13 Risinger JI, Umar A, Boyer JC, et al. Microsatellite instability in gynecological sarcomas and in hMSH2 mutant uterine sarcoma cell lines defective in mismatch repair activity. Cancer Res 1995;55:5664-5669.
14 Taylor NP, Gibb RK, Powell MA, et al. Defective DNA mismatch repair and XRCC2 mutation in uterine carcinosarcomas. Gynecol Oncol 2006;100:107-110.

15 Kowalski LD, Mutch DG, Herzog TJ, et al. Mutational analysis of MLH1 and MSH2 in 25 prospectivelyacquired RER+ endometrial cancers. Genes Chromosomes Cancer 1997;18:219-227.

16 Peiffer SL, Herzog TJ, Tribune DJ, et al. Allelic loss of sequences from the long arm of chromosome 10 and replication errors in endometrial cancers. Cancer Res 1995;55:1922-1926.

17 Boland CR, Thibodeau SN, Hamilton SR, et al. A National Cancer Institute Workshop on Microsatellite Instability for cancer detection and familial predisposition: development of international criteria for the determination of microsatellite instability in colorectal cancer. Cancer Res 1998;58:5248-5257.

18 Buttin BM, Powell MA, Mutch DG, et al. Increased risk for hereditary nonpolyposis colorectal cancer-associated synchronous and metachronous malignancies in patients with microsatellite instability-positive endometrial carcinoma lacking MLH1 promoter methylation. Clin Cancer Res 2004;10:481-490.

19 Szukala SA, Marks JR, Burchette JL, et al. Co-expression of p53 by epithelial and stromal elements in carcinosarcoma of the female genital tract: an immunohistochemical study of 19 cases. Int J Gynecol Cancer 1999;9:131-136.

20 Abargel A, Avinoach I, Kravtsov V, et al. Expression of p27 and p53: comparative analysis of uterine carcinosarcoma and endometrial carcinoma. Int J Gynecol Cancer 2004;14:354-359.

21 Pijnenborg JM, van de Broek L, Dam de Veen GC, et al. TP53 overexpression in recurrent endometrial carcinoma. Gynecol Oncol 2006;100:397-404.

22 Mariani A, Sebo TJ, Katzmann JA, et al. Endometrial cancer: can nodal status be predicted with curettage? Gynecol Oncol 2005;96:594-600. 